\title{
Morbimortalidad de la terapia endovascular en el tratamiento de estenosis carotídea extracraneana. Colombia 1996 - 2008
}

\author{
Sergio Vargas Vélez S. ${ }^{1}$, Jorge Múnera Chavarriaga ${ }^{2}$, \\ Claudia Patricia Zuluaga Gómez ${ }^{3}$
}

\section{Resumen}

Para el tratamiento de la enfermedad carotídea se recomienda como primera elección la endarterectomía, reservándose la angioplastia stent para pacientes con riesgo quirúrgico, por sus comorbilidades o por la localización de la estenosis.

Objetivo: Evaluar la morbimortalidad y los beneficios de la terapia endovascular: angioplastia y stent, para el tratamiento de la estenosis carotídea en pacientes tratados, durante un período de 12 años.

Materiales y métodos: Entre septiembre de 1996 y mayo de 2008 se realizaron 174 angioplastias e implantación de stent en 148 pacientes ( 26 de los cuales fueron tratados por estenosis bilateral).

Resultados y discusión: Los resultados mostraron una morbimortalidad de $2 \%$ que es menor o igual a lo reportado en la literatura mundial.

Palabras clave: estenosis carotídea, angioplastia, morbilidad, mortalidad.

\section{Title}

Morbimortality of endovascular therapy in the treatment of extracraneal carotid artery stenosis. Colombia 1996-2008.

\begin{abstract}
For the treatment of the carotid disease the endarterectomy is recommended as the first election, saving the angioplasty stent for patients with surgical risk, due to comorbidities or the location of the stenosis.
\end{abstract}

1 Neurorradiólogo y neurointervencionista del Instituto Nacional de Neurología y Neurocirugía Manuel Velasco Suárez, México (INNN), Jefe Posgrado Neurorradiología de la Universidad de Antioquia. Medellín, Colombia.

2 Neurorradiólogo de la Universidad de Antioquia. Medellín, Colombia.

3 Residente de Radiología, IV año de la Pontificia Universidad Javeriana - Hospital Universitario San Ignacio. 


\begin{abstract}
Objetive: To evaluate the morbidity, mortality and benefits of endovascular therapy: angioplasty and stenting for the treatment of carotid stenosis in patients treated during a period of 12 years.
\end{abstract}

\begin{abstract}
Materials and methods: Between september 1996 and may 2008, 174 angioplasties and implantation of stent were realized in 148 patients ( 26 of which were treated for bilateral stenosis).
\end{abstract}

Results and discussion: The results showed a mortality of $2 \%$ that is less or equal to the reported in the world literature.

Key words: carotid artery stenosis, angioplasty, morbility, mortality.

\section{Introducción}

Anualmente en los Estados Unidos se reportan 700.000 casos nuevos de accidentes cerebrovasculares (ACV), lo que constituye la tercera causa de muerte en dicho país después de las enfermedades cardiacas y del cáncer[1]. En Europa se calcula una incidencia global de 235/100.000, es decir, 1.000.000 nuevos casos por año[2] y en Shanghai, se reporta una incidencia de 76.1/100.000[3]. En Colombia se encontró una incidencia anual de 88.9/100.000[4], con una prevalencia nacional en mayores de 50 años de 19.9/1000[5].

El $80 \%$ de los ACV son isquémicos y de éstos entre el 20 y $30 \%$ son secundarios a enfermedad carotídea[1].

Tradicionalmente el manejo de la enfermedad carotídea sintomática depende del grado de estenosis que se presente. De acuerdo a esto, son de tratamiento médico las estenosis menores del $69 \%$ y para las estenosis mayores del $70 \%$ el manejo recomendado es el quirúrgico[1].
La terapia endovascular (mínimamente invasiva) empleada para el tratamiento de la enfermedad oclusiva carotídea extracraneana, ha generado interés en estas técnicas como alternativa a la revascularización quirúrgica[6-8]. El potencial de la angioplastia con balón y stent de la arteria carótida, para disminuir la incomodidad del paciente y las complicaciones secundarias al procedimiento quirúrgico, han hecho de ella una alternativa terapéutica viable en enfermedades como la estenosis aterosclerótica, la disección, el daño traumático y la displasia fibromuscular entre otras[9].

La expansión de esta técnica ha sido facilitada por varias causas tales como: el progreso de la tecnología en los equipos, los cuales, causan una menor radiación y dan una mayor resolución (angiógrafos rotacionales), la administración de fármacos con fines anestésicos para el procedimiento (neuroleptoanalgesia), el mejoramiento en el diseño de los dispositivos utilizados en el procedimiento de endoprótesis vascular (stent, balón), la disponibilidad de nuevos medicamen- 
tos antiagregantes, los cuidados posoperatorios con corto tiempo de recuperación (12 horas en promedio) y el entendimiento de la importancia en el manejo y prevención de las complicaciones endovasculares de los pacientes, han tenido indudablemente un gran impacto en el tratamiento de esta enfermedad por medio de esta técnica alternativa.

El tratamiento de la enfermedad carotídea con angioplastia y stent, está reservado para pacientes de alto riesgo quirúrgico. Pese a que en diferentes estudios se ha encontrado que la morbimortalidad entre ambos métodos terapéuticos (cirugía vs. terapia endovascular) es muy similar, dichos estudios muestran que es preferible el manejo quirúrgico.

Diferentes estudios como NASCET (The North American Symptomatic Carotid Endarterectomy Trial - Ensayo de Endarterectomía Carotídea Sintomática de Norte América), ECST (European Carotid Surgery Trial Ensayo Europeo de Cirugía de Carótida), y el Programa de Estudio Cooperativo de Asuntos de los Veteranos (Veterans Affairs Cooperative Study Program), fueron fundamentales para demostrar la efectividad de la revascularización quirúrgica en el tratamiento de la enfermedad carotídea oclusiva sintomática. Los pacientes incluidos en estos estudios tuvieron un episodio isquémico transitorio o un
ACV no incapacitante en los 6 meses precedentes y una estenosis carotídea ipsilateral de alto grado.

En NASCET la tabla de estimativo de sobrevida de cualquier ACV ipsilateral para 659 pacientes, en dos años, fue $26 \%$ en el grupo médico $(\mathrm{n}=331)$ y $9 \%$ en el grupo quirúrgico $(\mathrm{n}=328)$ con una reducción del riesgo absoluto (ARR) del 17\% ( $\mathrm{P}<.001)$. El número necesario a tratar (NNT) para prevenir un ACV anualmente, fue 12. En ECST, el riesgo de cualquier ACV ipsilateral para 778 pacientes en tres años fue $16,8 \%$ en el grupo médico $(n=323)$ y $2,8 \%$ en el grupo quirúrgico $(n=455)$, con un ARR de $14 \%(\mathrm{P}<.0001)$. El NNT para prevenir un ACV anualmente fue $21[9,7,10-12]$.

CAVATAS (The Carotid and Vertebral Artery Transluminal Angioplasty Study - Estudio de Angioplastia Trasluminal de la Arteria Carótida y Vertebral) fue un estudio multicéntrico con asignación aleatoria realizado en 504 pacientes tratados con angioplastia carotídea, (con y sin stent) o cirugía. Todos los pacientes fueron considerados candidatos para cirugía o terapia endovascular y la mayoría (90\%) tenían síntomas referidos a la arteria tratada en un período de 6 meses antes. No se encontró una diferencia significativa en el riesgo de ACV o sobrevida entre los grupos de tratamiento a los 30 días[6, 9, 13-17]. 
Varios estudios diseñados para evaluar la seguridad y eficacia de la angioplastia-stent carotídeo (carotid angioplasty and stenting, CAS) se están llevando a cabo y continúan inscribiendo pacientes. CREST (Carotid Revascularization Endarterectomy versus Stenting Trial - Endarterectomía de Revascularización Carotídea versus Estudios de Stents), es un estudio aleatorizado prospectivo y multicéntrico que está actualmente inscribiendo pacientes. Inicialmente diseñado para comparar $C A S$ con $C E A$ en pacientes que tenían enfermedad sintomática y estenosis carotídea $(=50 \%$ por angiografía o $=70 \%$ por doppler), el grupo fue ampliado a los pacientes asintomáticos con estenosis carotídea $(=60 \%$ por angiografía o $=70 \%$ por doppler). Igualmente existen otros estudios de seguimiento en diferentes países [9, 18, 19].

Actualmente $C A S$ es usado para tratamiento de pacientes que tienen circunstancias a favor de un tratamiento endovascular. Éstas son: abordaje quirúrgico con dificultades técnicas (bifurcación carotídea alta por ejemplo), condiciones médicas de comorbilidad, enfermedad oclusiva inducida por radiación y estenosis después de cirugía.

Adicionalmente $C A S$ es usado en casos seleccionados de displasia fibromuscular, arteritis de Takayasu o después de disección arterial. CAS no es considerada inferior a la endarte- rectomía en las mencionadas situaciones clínicas y puede ser considerada una alternativa terapéutica a la cirugía cuando es realizada por operadores que han establecido una morbilidad y mortalidad perioperatoria de 4 a $6 \%$ [ 6, 17].

Las limitaciones de la $C A S$ son la contraindicación de la angiografía, la imposibilidad de acceder a la carótida por oclusión de los accesos vasculares, las enfermedades del colágeno, la presencia de elongaciones y los bucles, que hacen difícil la navegación del stent.[ 20].

En nuestro medio no se dispone de centros de referencia para el tratamiento quirúrgico de dicha enfermedad, siendo la terapia endovascular una alternativa de tratamiento, que contribuye a tener índices estadísticos de morbimortalidad similares a los reportados en la literatura mundial.

\section{Materiales y métodos}

El presente estudio es retrospectivo, observacional, descriptivo, de una serie de casos.

Incluye los pacientes tratados, durante el periodo comprendido entre septiembre de 1996 y mayo de 2008.

En este estudio se llevaron a cabo 174 procedimientos en 148 pacientes $\mathrm{y}$ en diferentes instituciones del país: 
Hospital Universitario San Vicente de Paúl, Instituto Cardiovascular y de Estudios Especiales INCARE Clínica Las Vegas y, Clínica Las Américas (Medellín); Hospital Santa Sofía (Manizales); Clínica AMI (Cartagena) y Clínica Central el Quindío (Armenia).

Como fuente de información se utilizó la base de datos del médico tratante con los estudios angiográficos realizados a los pacientes remitidos con diagnóstico de estenosis carotídea y tratados con terapia endovascular. Sus criterios de inclusión fueron: pacientes que cumplían con los criterios NASCET, estenosis mayores del $60 \%$, pacientes sintomáticos en los últimos 180 días antes del procedi- miento, y pacientes asintomáticos de alto riesgo).

En la etapa inicial se describieron los datos demográficos así como la localización y el porcentaje de la estenosis. Además, se calculó el porcentaje de complicaciones con el fin de comparar los datos obtenidos con los descritos en otras publicaciones internacionales.

\section{Procedimiento}

Todos los procedimientos fueron realizados en salas de Neuroangiografía, mediante las técnicas de sustracción digital y road mapping, con anestesia local y sedación consciente. Figuras 1 y 2.

\section{a. Balón coronario, b. Protección, c. Stent autoexpandible, d. Angioplastia.}

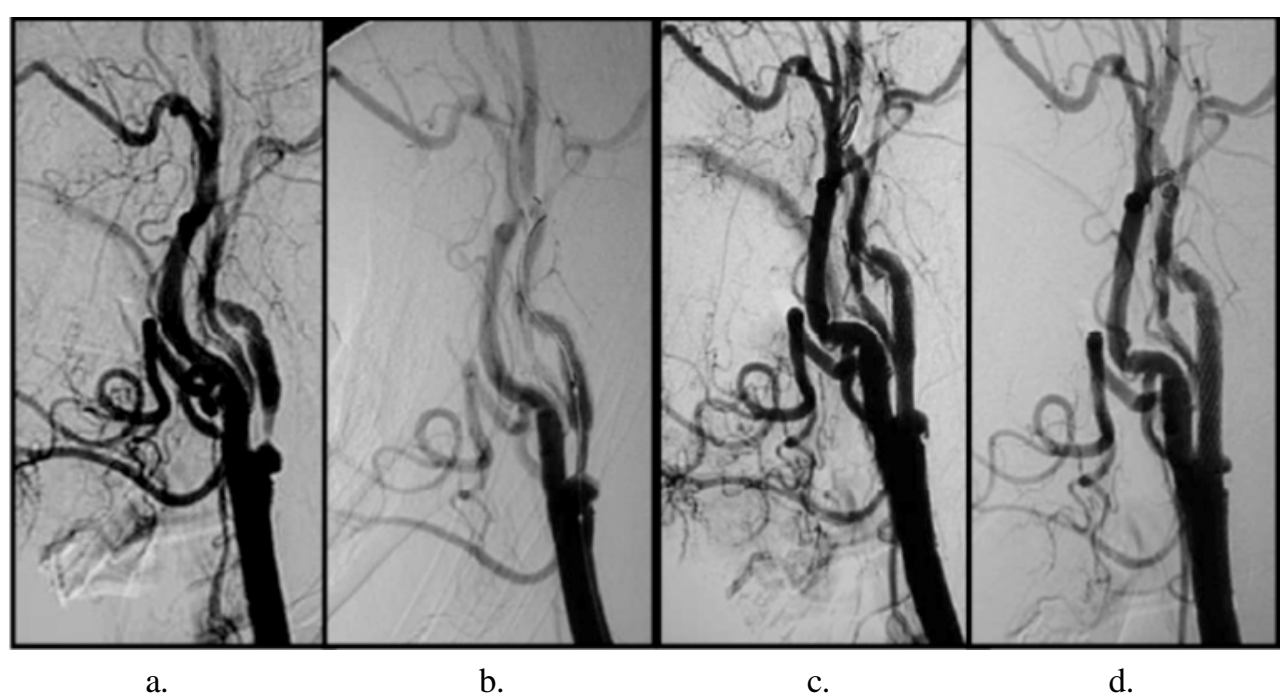

Figura 1. Pasos para la realización de angioplastia -stent. 
a. Antes, b. Después.

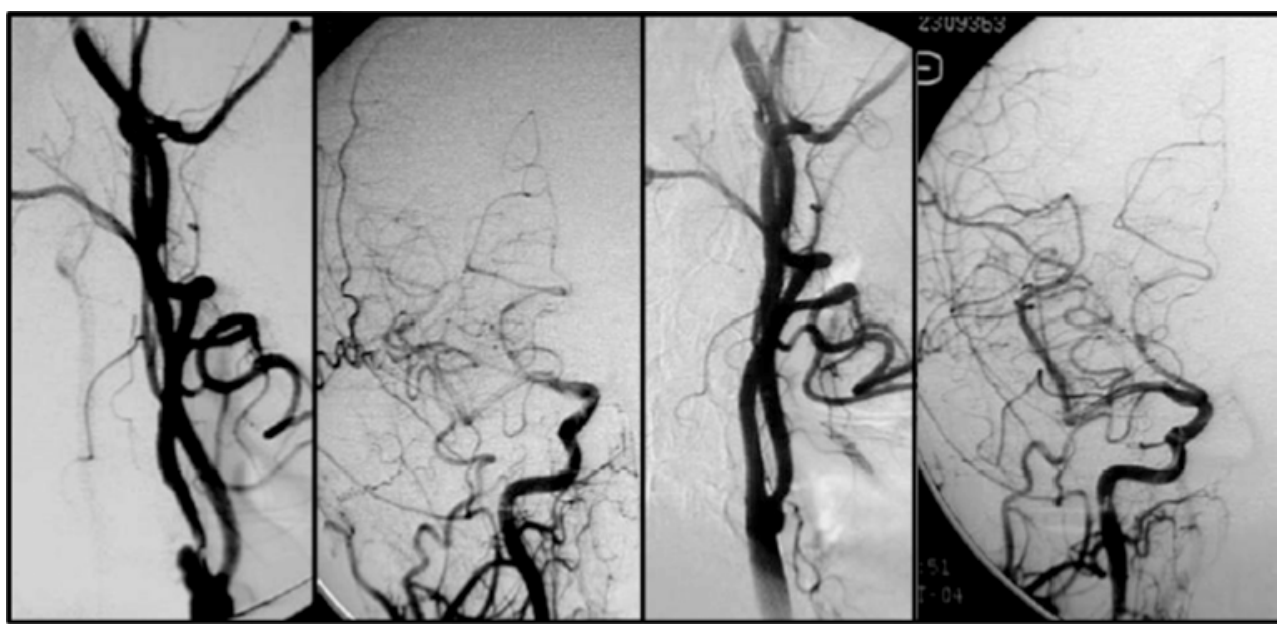

a.

b.

Figuura 2. Cambio en la perfusión cerebral después de la angioplastia-stent.

Para los pacientes tratados entre 1996 y 2002, se realizó antiagregación previa con ticlopidina y aspirina. Posterior a esta fecha se empleó el clopidogrel (75 miligramos/día) y aspirina (100 miligramos/día) tres días antes de la intervención. Se utilizó introductor femoral largo 6fr., guiado con guía y catéter, y 5000 unidades de heparina intravenosa durante el procedimiento. A partir del año 2002 se utilizaron sistemas de protección distal para evitar tromboembolia. El stent utilizado fue Wallstent con dimensiones de 7 x 30 y se hizo control angiográfico al terminar el procedimiento.

A todos los pacientes se les formuló terapia de antiagregación con clopidogrel (75 miligramos/día), du- rante 6 meses y aspirina (100 miligramos/día) indefinidamente.

Se les realizó seguimiento a todos los pacientes, mediante doppler color a los 6 y 12 meses y luego anualmente.

Se firmaron consentimientos escritos por parte de todos los pacientes tratados, previa información del procedimiento y posibles complicaciones.

\section{Resultados}

Fueron realizados 174 procedimientos en 148 pacientes, 26 pacientes fueron tratados bilateralmente, 67 mujeres y 81 hombres. Figura 3. Los pacientes se encontraban entre los 14 y 92 años con una media de 69.38 . 


\section{GENERO}

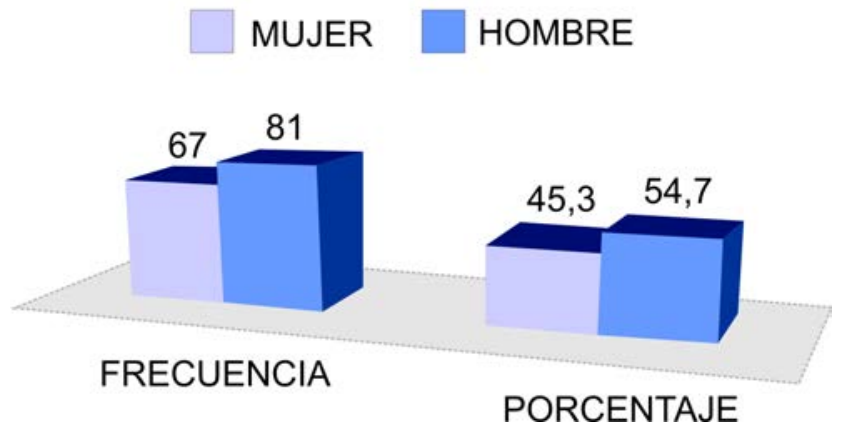

Figura 3

\section{PORCENTAJE DE ESTENOSIS}

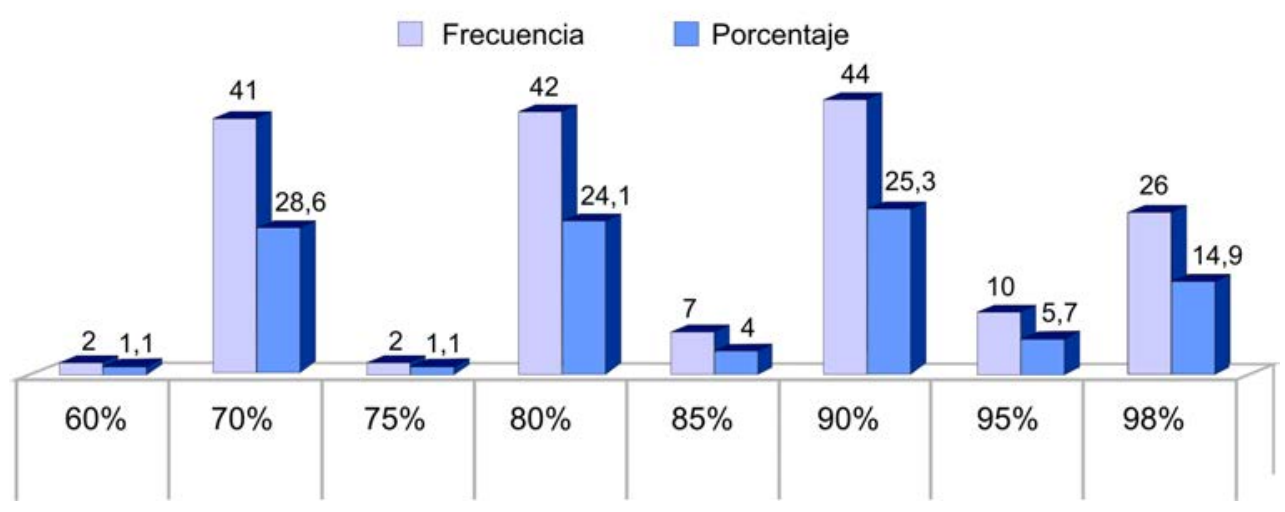

Figura 4

Causas de Estenosis Carotídea encontradas en este estudio: 131 pacientes fueron tratados por enfermedad arteriosclerótica carotídea, 8 pacientes por estenosis secundarias a radioterapia, 5 pacientes por estenosis posquirúrgica, 3 pacientes por enfermedad de Takayasu y 2 por disección no traumática.
Se encontraron 13 pacientes con una oclusión del $100 \%$ de la arteria contralateral. Figura 4.

Intervenciones vasculares concomitantes, se realizaron en 12 pacientes así: en 9 de ellos se colocaron stent en otras arterias (2 stent renales, 1 stent iliaco bilateral y 6 stent de subclavia). Figura 5. 


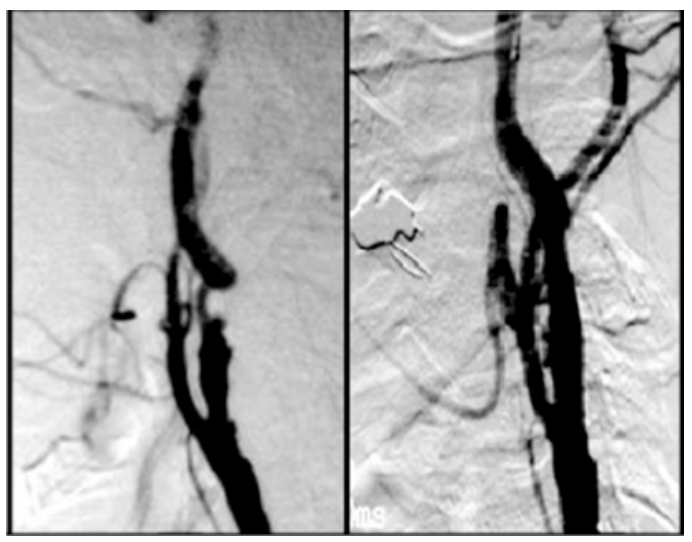

a.

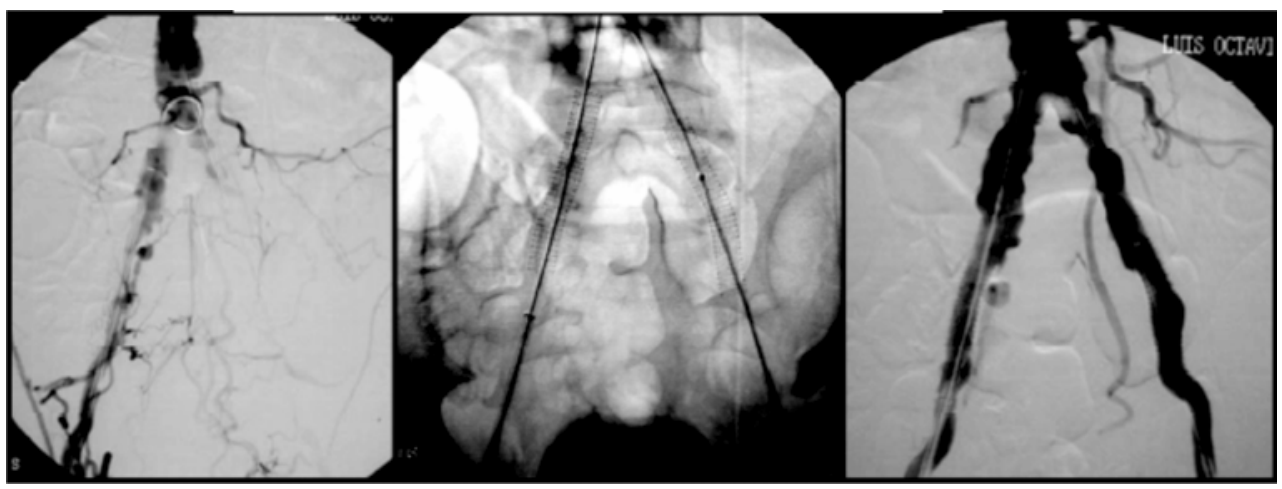

b.

Figura 5. Paciente tratado simultáneamente, a. estenosis carotídea b. estonosis de arterias iliacas.

Tres pacientes con aneurismas intracraneanos se trataron en el mismo procedimiento ( 1 de arteria comunicante anterior, 1 carotido-oftálmico y 1 de cerebral media).

Durante la angiografía diagnóstica se encontraron 6 pacientes con placas ulceradas y un paciente con trombo intraluminal.

Se estudiaron 5 pacientes con enfermedad de 3 vasos coronarios de los cuales 2 fueron tratados por estenosis carotídea bilateral. Hubo 1 procedimiento fallido por tortuosidad de la aorta.

En el seguimiento de los pacientes tratados, se encontró que 4 de ellos (3\%) desarrollaron hiperplasia intimal.

Fallecieron 3 pacientes (2\%): 1 por hiperperfusión y los otros 2 por insuficiencia cardiaca congestiva (enfermedad de 3 vasos coronarios). Figura 6. 


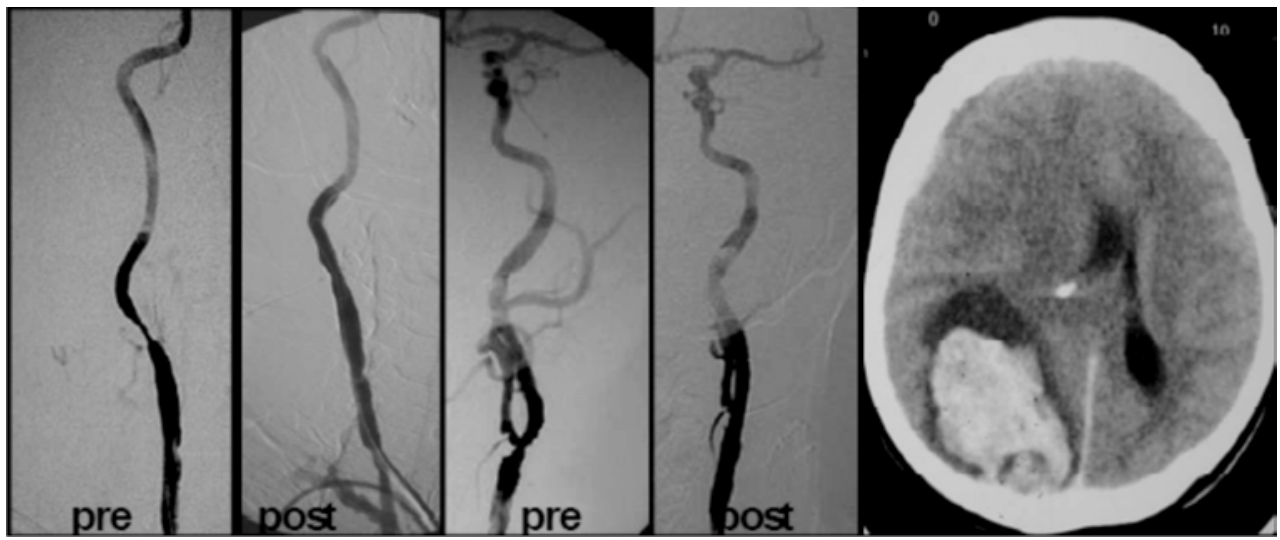

Figura 6. Paciente tratado de estenosis carotídea bilateral, desarrolla síndrome de hiperperfusión, fallece.

\section{Discusión}

De los 148 pacientes a quienes se les practicó el procedimiento, sólo en un caso éste fue fallido.

Un paciente (1\%), presentó un episodio de isquemia cerebral transitoria (ICT), del que se recuperó totalmente a los cinco minutos y ocurrió en el período antes de que se usara el dispositivo de protección distal.

Se registraron tres (3) muertes (2\%), entre los pacientes a quienes se les realizó el procedimiento, estando esto acorde con los reportes de otros estudios. Se presentaron 4 casos de hiperplasia intimal ( $3 \%$ de los pacientes, $2 \%$ de los procedimientos), tres sintomáticos, a los cuales se les practicó nueva angioplastia y uno requirió colocación de stent. Estos porcentajes son similares con los reportados en otros estudios que oscilan entre el 2 y $5 \%$, CAVATAS reportó un $14 \%$.

\section{Conclusiones}

- La angioplastia e implantación de stent carotídeo extracerebral como tratamiento de la estenosis carotídea de múltiples causas, es una modalidad terapéutica reproducible, con excelentes resultados y con una muy baja morbimortalidad.

- Este procedimiento en manos de personal adecuadamente entrenado, sumado a los desarrollos actuales de los stent, catéteres, guías, sistemas de protección distal, y equipos rotacionales de angiografía, es un procedimiento de corta duración, seguro, no requiere anestesia general y su posoperatorio produce muy pocas molestias al paciente. Requiere una muy breve hospitalización para su recuperación.

- La terapia endovascular tiene la ventaja de poder tratar en el mismo acto, enfermedad de varios vasos. 
- Los pacientes con enfermedad de tres vasos y enfermedad carotídea, que van a ser llevados a cirugía cardiaca, presentan una buena evolución posterapia endovascular garantizándoles una adecuada perfusión cerebral en el momento de la cirugía disminuyendo así el riesgo de ACV por hipotensión.

\section{Bibliografía}

1. Faries PL, Chaer RA, Patel S, DeRubertis B, Lin SC, Kent CK. Current Management of Extracranial Carotid Artery Disease. Vascular and Endovascular Surgery. 2006;40:165-75.

2. Truelsen T, Ekman M, Boysen G. Cost of stroke in Europe. European Journal of Neurology. 2005;12:78-84.

3. Jiang B, Wang WZ, Chen H, Hong Z, Yang QD, Wu SP, et al. Incidence and trends os stroke and its subtypes in China. Results from three large cities. Stroke. 2006;37:63-8.

4. Uribe CS, Jiménez I, Mora MO, Arana A, Sánchez JL, Zuluaga L, et al. Epidemiología de las enfermedades cerebrovasculares en Sabaneta, Colombia (1992- 1993). Revista de Neurología. 1997;25:1008-12.

5. Pradilla G, Vesga B, León FE y Grupo GENECO. Estudio neuroepidemiológico nacional (EPINEURO) colombiano. Revista Panamericana de Salud Pública. 2003;14;104-11.

6. Cremonesi A, Setacci C, Bignamini A, Bolognese L, Briganti F, Di Sciascio $\mathrm{G}$, et al. Carotid Artery Stenting First
Consensus Document of the ICCSSPREAD Joint Committee. Stroke. 2006;37:2400-9.

7. A Report of the SCAI/SVMB/SVS Writing Committee to Develop a Clinical Competence Statement on Carotid Interventions. Clinical Competence Statement on Carotid Stenting: Training and Credentialing for Carotid Stenting - Multispecialty Consensus Recommendations. Catheterization and Cardiovascular Interventions. 2005;64:1-11.

8. Levy E, Ecker R, Thompson J, Toward clinical equipoise: the current case for carotid angioplasty and stent placement. Neurosurgical Focus. 2005;18:e3.

9. Barrett KM, Brott TG. Carotid Artery Stenting Versus Carotid Endarterectomy: Current Status. Neurologic Clinics. 2006;24:681-95.

10. Chaturvedi S, Bruno A, Feasby T, Cohen SN, Cote R, Hess D et al. Carotid endarterectomy-An evidence-based review: Report of the Therapeutics and Technology Assessment Subcommittee of the American Academy of Neurology. Neurology. 2005;65:794-801.

11. Morris, P. Angioplasty and Stenting. Practical Neuroangiography. Second edition. Philadelphia: Lippincott Williams \& Wilkins. 2007;506-24.

12. Lahoz C, Mostaza JM. La ateroesclerosis como enfermedad sistémica. Revista. Española de Cardiología. 2007; 60:184-95.

13. Mas J, Chatellier G, Beyssen B, Becquemin JP, Larrue V, Lièvre $\mathrm{M}$ et al. Endarterectomy versus Stenting in 
Patients with Symptomatic Severe Carotid Stenosis. New England Journal of Medicine. 2006;355:1660-71.

14. Shrivastava V, Sookhoo S, Macdonald Dunning J. Is carotid artery stenting equivalent or superior to carotid endarterectomy for treatment of carotidartery stenosis? Interact. Cardiovascular Thoracic Surgery. 2005; 4:550-4.

15. Coward L, Featherstone R, Brown M. Safety and Efficacy of Endovascular Treatment of Carotid Artery Stenosis Compared With Carotid Endarterectomy. Stroke. 2005;36:905-11.

16. Das S, Bendok B, Getch C. Update on current registries and trials of carotid artery angioplasty and stent placement. Neurosurgical Focus. 2005;18:(1)e2.
17. Hacke W. 30 day results from the SPACE trial of stent-protected angioplasty versus carotid endarterectomy in symptomatic patients: a randomised non - inferiority trial. Lancet. 2006;368:1239-47.

18. Qureshi A. Endovascular treatment of cerebrovascular diseases and intracranial neoplasms. Lancet. 2004;363:804-13.

19. Cullen S, Higashida R. Endovascular treatment of extracranial carotid Artery stenosis: update on carotid angioplasty and steting. Neuroimag Clinics of North America. 2005;15:383-95.

20. Castro E, González A. Tratamiento endovascular de la enfermedad carotídea. Situación actual, aspectos técnicos y capacitación profesional. Revista de Neurología. 2005;41:749-56. 